Pacific Journal of Mathematics

THE SELBERG TRACE FORMULA FOR GROUPS WITHOUT 


\title{
THE SELBERG TRACE FORMULA FOR GROUPS WITHOUT EISENSTEIN SERIES
}

\author{
PAUl F. Ringseth
}

\begin{abstract}
Let $G$ be a reductive Lie group, $\Gamma$ a nonuniform lattice in $G$. Let $\chi$ be a finite dimensional unitary representation of $\Gamma$. In order to have Eisenstein series, $(G, \Gamma)$ must satisfy a certain assumption. The purpose of this note is to compute the Selberg trace formula for pairs $(G, \Gamma)$ that do not possess Eisenstein series. A necessary preliminary to this, is a trace formula for $\operatorname{Ind}_{\Gamma}^{G}(\chi)$. This is also presented.
\end{abstract}

Introduction. Let $G$ be a reductive Lie group of the Harish-Chandra class; let $\Gamma$ be a nonuniform lattice in $G$. Let $\chi$ be a finite dimensional unitary representation of $\Gamma$. Denote by $L^{2}(G / \Gamma ; \chi)$ the representation space of $\operatorname{Ind}_{\Gamma}^{G}(\chi)$-then $G$ acts on $L^{2}(G / \Gamma ; \chi)$ via the left regular representation $L_{G / \Gamma}$. Let $L_{G / \Gamma}^{\text {dis }}$ be the restriction of $L_{G / \Gamma}$ to $L_{\text {dis }}^{2}(G / \Gamma ; \chi)-$ the maximal completely reducible subspace. One of the central problems in the theory of automorphic forms is computing the trace of $L_{G / \Gamma}^{\text {dis }}(\alpha)\left(\alpha \in C_{c}^{\infty}(G)\right)$; viz. the Selberg trace formula.

Let $L_{\text {con }}^{2}(G / \Gamma ; \chi)$ be the orthogonal complement of $L_{\text {dis }}^{2}(G / \Gamma ; \chi)$ in $L^{2}(G / \Gamma ; \chi)$ and let $L_{G / \Gamma}^{\text {con }}$ be the corresponding representation-then most attacks on the Selberg trace formula begin by expressing the integral kernel of $L_{G / \Gamma}^{\text {con }}(\alpha)\left(\alpha \in C_{c}^{\infty}(G)\right)$ in terms of Eisenstein series. However, a certain assumption (cf. p. 16 of [L2] and p. 62 of [OW1]) needs to be satisfied by the pair $(G, \Gamma)$ in order for a satisfactory theory of Eisenstein series to exist. The purpose of this note is to compute the Selberg trace formula for pairs $(G, \Gamma)$ without Eisenstein series; i.e. that do not satisfy the assumption supra.

In order to accomplish this a trace formula needs to be given for $L_{\text {dis }}^{2}(G / \Gamma ; \chi)$, when $\chi \neq 1$. This has been done in the case $G=\mathrm{SL}_{2}(\mathbf{R})$ by Venkov (cf. [V1]). Moore has also done preliminary work for the real rank one situation (cf. [M1]). For the general case, Eisenstein series need to be defined with respect to $\chi$ and a spectral decomposition following Langlands needs to be given. This was accomplished by the author in his thesis (cf. [R1]).

When $(G, \Gamma)$ does not possess Eisenstein series, the procedure to compute the trace formula is to describe $L_{G / \Gamma}$ in terms of the left 
regular representation of $L^{2}\left(G_{n} / \Gamma_{n} ; \chi_{n}\right)$, where the pair $\left(G_{n}, \Gamma_{n}\right)$ is canonically constructed from $(G, \Gamma)$ and does possess Eisenstein series. It should be noted that, in general, $\chi_{n}$ will be non-trivial, even when $\chi=1$. This is carried out in $\S 2$.

Section 1 is comprised of summarizing the facts needed about the spectral decomposition of $L^{2}(G / \Gamma ; \chi)$, in order to describe the integral kernel of $L_{G / \Gamma}^{\text {con }}$ in terms of Eisenstein series.

A new type of truncation operator, due to Müller is introduced in $\S 3$ and the effect of truncating the kernels is computed (cf. [MU1]).

The trace formula presented in $\S 4$ follows the work of Osborne and Warner in [OW2] and uses the truncation operator of Müller to simplify the Dini calculus.

I would like to thank Osborne and Warner for suggesting this problem and for the substantial help they gave me in completing the spectral decomposition of $L^{2}(G / \Gamma ; \chi)$.

1. Preliminaries. (1) Let $G$ be a reductive Lie group of the HarishChandra class; let $\Gamma$ be a nonuniform lattice in $G$. Assume that the pair satisfies the assumption spelled out on page 62 of [OW1] or equivalently the assumption on page 16 of [L2]. Let $(\chi, V)$ be a finite dimensional unitary representation of $\Gamma$. Denote by $L^{2}(G / \Gamma ; \chi)$ the representation space of the corresponding induced representation $\operatorname{Ind}_{\Gamma}^{G}(\chi)$. Following Langlands [cf. [L1], [L2] and [OW1]], the author has obtained the spectral decomposition of the left regular representation $L_{G / \Gamma}$ acting on $L^{2}(G / \Gamma ; \chi)$, in terms of principal series representations of $G$ [cf. [R1]].

Denote by $L_{G / \Gamma}^{\text {dis }}$ the subrepresentation of $L_{G / \Gamma}$, acting on the maximal completely reducible subspace $L_{\text {dis }}^{2}(G / \Gamma ; \chi)$. There is then an orthogonal decomposition

$$
L^{2}(G / \Gamma ; \chi)=L_{\mathrm{dis}}^{2}(G / \Gamma ; \chi) \oplus L_{\mathrm{con}}^{2}(G / \Gamma ; \chi) .
$$

Let $\alpha \in C_{c}^{\infty}(G)$. Let $K_{\alpha}(x, y)$ denote the integral kernel of $L_{G / \Gamma}(\alpha)-$ then, with respect to the decomposition supra, there are integral kernels

$$
\left\{\begin{array}{l}
K_{\alpha}^{\mathrm{dis}}(x, y), \\
K_{\alpha}^{\operatorname{con}}(x, y),
\end{array}\right.
$$

corresponding to the representations

$$
\left\{\begin{array}{l}
L_{G / \Gamma}^{\mathrm{dis}}(\alpha), \\
L_{G / \Gamma}^{\text {con }}(\alpha) .
\end{array}\right.
$$


In order to compute the trace of $L_{G / \Gamma}^{\mathrm{dis}}(\alpha)$, it is more convenient to work with

$$
K_{\alpha}(x, y)-K_{\alpha}^{\mathrm{con}}(x, y) .
$$

Hence, we shall need to recall the description of $K_{\alpha}^{\text {con }}(x, y)$ in terms of Eisenstein series.

(2) A maximal compact subgroup $K$ of $G$ has been fixed. Let $\delta$ belong to the unitary dual $\hat{K}$ of $K$. Let $P$ be a ( $\Gamma$-cuspidal) parabolic subgroup of $G$, with Langlands decomposition $P=M \cdot A \cdot N$. We shall always assume that $A$ is stable under the Cartan involution. Denote by $\mathscr{O}$, the orbit of an infinitesimal character of $M$ under the action of the "Weyl group" $W(A) . \quad(W(A)$ consists of all automorphisms of $A$ induced by an inner automorphism of $G$.) There is a natural representation $\chi_{P}$ of

$$
\Gamma_{M}=\Gamma \cap P / \Gamma \cap N
$$

on

$$
V_{P}=\{v \in V \mid \chi(\Gamma \cap N) v=v\} .
$$

Let $\operatorname{pr}_{P}$ be the orthogonal projection of $V$ onto $V_{P}$. Define

$$
\mathscr{E}_{\text {dis }}\left(\delta, \mathscr{O} ; \chi_{P}\right)
$$

to be the space of $V_{P}$-valued square integrable automorphic forms on $G / A N$, with $K$-type $\delta$ and orbit type $\mathscr{O}$, that transform on the right according to $\chi_{P}$. This forms a finite dimensional subspace of

$$
L^{2}\left(K \times M / \Gamma_{M} ; \chi_{P}\right) .
$$

Let $\delta \in \hat{K}$-then define $\xi_{\delta}$ to be the normalized character of $\delta$. Let $\mathscr{F}$ be the collection of finite subsets of $\hat{K}$ ordered by inclusion. Let $F \in \mathscr{F}$. Denote by $C_{c}^{\infty}(G ; F)$ the set of $f \in C_{c}^{\infty}(G)$ such that

$$
\bar{\xi}_{\delta} * f * \bar{\xi}_{\delta}=f
$$

for all $\delta \in F$. Define

$$
C_{c}^{\infty}(G ; K)=\underset{\mathscr{F}}{\lim } C_{c}^{\infty}(G ; F) .
$$

Then $C_{c}^{\infty}(G ; K)$ is an $L F$-space, consisting of all $K$-finite elements of $C_{c}^{\infty}(G)$, whose topology is finer than the subspace topology of $C_{c}^{\infty}(G)$.

Denote by $L_{\text {loc }}^{2}(G / \Gamma ; \chi)$ the space of all measurable functions

$$
\left\{\begin{array}{l}
f: G \rightarrow V, \\
f(x \gamma)=\chi\left(\gamma^{-1}\right) f(x) \quad(\gamma \in \Gamma, x \in G),
\end{array}\right.
$$


such that $\|f(\cdot)\|$ is locally integrable on $G / \Gamma$. Let $f \in L_{\text {loc }}^{1}(G / \Gamma ; \chi)-$ then

$$
f^{P}(x)=\int_{N / N \cap \Gamma} \operatorname{pr}_{P} f(x n) d n
$$

is called the constant term of $f$ along $P$. If $f^{P}=0$ for all $P \neq G$, then $f$ is called a cusp form on $G$. Denote by

$$
L_{\text {cus }}^{2}(G / \Gamma ; \chi)
$$

the space of square integrable cusp forms - then there is an orthogonal decomposition

$$
L_{\text {dis }}^{2}(G / \Gamma ; \chi)=L_{\text {cus }}^{2}(G / \Gamma ; \chi) \oplus L_{\text {res }}^{2}(G / \Gamma ; \chi) .
$$

The subspace $L_{\mathrm{res}}^{2}(G / \Gamma ; \chi)$ is called the residual spectrum and is spanned by the residues of Eisenstein series associated with cusp forms [cf. $\S 7$ of [L2]].

Let $x \in G$-then $x=k m a n$, where $k \in K, m \in M, a \in A$ and $n \in N$. The factor $a$ is uniquely determined by $x$ and the Langlands decomposition of $P$. Hence, for $\Lambda \in \check{\mathfrak{a}} \otimes \mathbf{C}$, set

$$
H_{P}(x)=\log (a)
$$

and

$$
\xi_{\Lambda}(x)=e^{\Lambda\left(H_{P}(x)\right)} .
$$

Two parabolic subgroups $P$ and $P^{\prime}$ of $G$ are said to be associate, if their split components $A$ and $A^{\prime}$ are $G$-conjugate. The space of such maps from $A$ to $A^{\prime}$ is denoted $W\left(A^{\prime}, A\right)$. Let $\mathscr{C}$ be a class of associate parabolic subgroups of $G$. If $\mathscr{C}^{*}$ is a subset of $\mathscr{C}$ comprised of $\Gamma$ conjugacy classes and $\mathscr{O}=\left\{\mathscr{O}_{P}\right\}_{P \in \mathscr{C}}$ is a collection of associate orbits, put

$$
\left\{\begin{array}{l}
\check{\mathfrak{a}}_{\mathscr{C}^{*}}=\left\{\Lambda \in \prod_{P \in \mathscr{C}^{*}} \check{\mathfrak{a}}_{P} \mid \Lambda_{x_{P}}=\operatorname{Ad}(x) \Lambda_{P}(x \in G)\right\}, \\
\mathfrak{a}_{\mathscr{C}^{*}}=\left\{\mathbf{H} \in \prod_{P \in \mathscr{C}^{*}} \mathfrak{a}_{P} \mid \mathbf{H}_{P}=\operatorname{Ad}\left(\gamma^{-1}\right) \mathbf{H}_{\gamma_{P}}+H_{P}(\gamma)(\gamma \in \Gamma)\right\}, \\
\mathscr{E}_{\text {dis }}\left(\delta, \mathscr{O} ; \mathscr{C}^{*}\right) \\
=\left\{\varphi \in \prod_{P \in \mathscr{C}^{*}} \mathscr{E}_{\text {dis }}\left(\delta, \mathscr{O}_{P} ; \chi_{P}\right) \mid \varphi_{\varphi_{P}}(x)=\chi(\gamma) \varphi_{P}(x \gamma)(\gamma \in \Gamma)\right\},
\end{array}\right.
$$

where ${ }^{\gamma} P=\gamma P y^{-1}$ and ${ }^{x} P=x P x^{-1}$. 
Fix once and for all an element $\mathbf{H} \in \mathfrak{a}_{\mathscr{C}}$. The Eisenstein series associated to an element $\varphi$ of $\mathscr{E}_{\text {dis }}\left(\delta, \mathscr{O} ; \mathscr{C}^{*}\right)$ is

$$
\mathbf{E}(\mathscr{C}: \varphi: \Lambda: x)=\sum_{P \in \mathscr{C}^{*}} \varphi_{P}(x) \cdot e^{\left\langle\Lambda_{P}-\rho_{P}, H_{P}(x)-\mathbf{H}_{P}\right\rangle}
$$

where the real part of $\Lambda$ is restricted to lie in some sector of $\check{\mathfrak{a}}_{\mathscr{C}}$ * to facilitate convergence. The Eisenstein series possesses a meromorphic continuation to all of $\check{\mathfrak{a}}_{\mathscr{C}} \otimes \mathbf{C}$.

The induced representations also make an appearance in this setting. One has a natural representation $\left(\mathscr{O}_{P}, \Lambda_{P}\right)$ of $P$ on $L_{\mathrm{dis}}^{2}(M / \Gamma ; \mathscr{O})$ :

$$
\left\{\begin{array}{l}
M \text { operates by the left regular representation, } \\
A \text { operates via multiplication by the quasi-character } \xi_{-\Lambda_{P}}, \\
N \text { operates trivially. }
\end{array}\right.
$$

Call

$$
\operatorname{Ind}_{P}^{G}\left(\mathscr{O}_{P}, \Lambda_{P}\right)
$$

the associated principal series representation of $G$. Let

$$
\operatorname{Ind}_{\mathscr{C}}^{G}(\mathscr{O}, \Lambda)
$$

denote the corresponding representation on

$$
\mathscr{E}_{\text {dis }}(\mathscr{O} ; \mathscr{C})=\sum_{\delta \in \hat{K}} \bigoplus \mathscr{E}_{\text {dis }}(\delta, \mathscr{O} ; \mathscr{C})
$$

However, the representation space of

$$
\operatorname{Ind}_{\mathscr{C}}^{G}(\mathscr{O}, \Lambda)
$$

shall be denoted by

$$
\mathscr{E}_{\text {dis }}(\mathscr{O} ; \Lambda)
$$

Let $\alpha \in C_{c}^{\infty}(G ; K)$-then

$$
\alpha * \mathbf{E}\left(\mathscr{C}^{*}: \varphi: \Lambda\right)=E\left(\mathscr{C}^{*}: \operatorname{Ind}_{\mathscr{C}}^{G}(\mathscr{O}, \Lambda)(\alpha) \varphi: \Lambda\right) .
$$

Let $\mathscr{C}_{i}$ and $\mathscr{C}_{j}$ be $G$-conjugacy classes occurring in $\mathscr{C}$-then there is a canonical intertwining operator

$$
\mathbf{c}_{\mathrm{dis}}\left(\mathscr{C}_{j} \mid \mathscr{C}_{i}: \mathbf{w}: \Lambda_{i}\right) \quad\left(\mathbf{w} \in \mathbf{W}\left(\mathscr{C}_{j}, \mathscr{C}_{i}\right)\right),
$$

characterized by the conditions

$$
\left\{\begin{array}{l}
\mathbf{E}\left(\mathscr{C}_{i}: \varphi_{i}: \Lambda_{i}\right)=\mathbf{E}\left(\mathscr{C}_{j}: \mathbf{c}_{\mathrm{dis}}\left(\mathscr{C}_{j} \mid \mathscr{C}_{i}: \mathbf{w}: \Lambda_{i}\right) \varphi_{i}: \mathbf{w} \Lambda_{i}\right), \\
\mathbf{c}_{\mathrm{dis}}\left(\mathscr{C}_{j} \mid \mathscr{C}_{i}: \mathbf{w}: \Lambda_{i}\right) \circ \mathbf{I n d}_{\mathscr{C}_{i}}^{G}\left(\mathscr{O}_{i}, \Lambda_{i}\right)(\alpha) \\
=\mathbf{I n d}_{\mathscr{C}_{j}}^{G}\left(\mathbf{w} \cdot \mathscr{O}_{i}, \mathbf{w} \Lambda_{i}\right)(\alpha) \circ \mathbf{c}_{\operatorname{dis}}\left(\mathscr{C}_{j} \mid \mathscr{C}_{i}: \mathbf{w}: \Lambda_{i}\right)\left(\alpha \in C_{c}^{\infty}(G ; K)\right),
\end{array}\right.
$$


and satisfying the functional equation

$$
\begin{gathered}
\mathbf{c}_{\mathrm{dis}}\left(\mathscr{C}_{k} \mid \mathscr{C}_{i}: w_{k j} w_{j i}: \Lambda_{i}\right) \quad\left(w_{k j} \in \mathbf{W}\left(\mathscr{C}_{k}, \mathscr{C}_{j}\right), w_{j i} \in \mathbf{W}\left(\mathscr{C}_{j}, \mathscr{C}_{i}\right)\right) \\
=\mathbf{c}_{\mathrm{dis}}\left(\mathscr{C}_{k} \mid \mathscr{C}_{j}: w_{k j}: w_{j i} \Lambda_{i}\right) \circ \mathbf{c}_{\mathrm{dis}}\left(\mathscr{C}_{j} \mid \mathscr{C}_{i}: w_{j i}: \Lambda_{i}\right) .
\end{gathered}
$$

In fact

$$
\mathbf{c}_{\mathrm{dis}}\left(\mathscr{C}_{j} \mid \mathscr{C}_{i}: \mathbf{w}: \Lambda_{i}\right)
$$

is a linear transformation from

$$
\mathscr{E}_{\text {dis }}\left(\delta, \mathscr{O}_{i} ; \mathscr{C}_{i}\right) \text { to } \mathscr{E}_{\text {dis }}\left(\delta, \mathbf{w} \cdot \mathscr{O}_{i} ; \mathscr{C}_{j}\right),
$$

that is meromorphic as a function of $\Lambda_{i}$ on $\mathfrak{a}_{\mathscr{C}_{i}} \otimes \mathbf{C}$. (Here $\mathscr{O}_{i}=$ $\left\{\mathscr{O}_{P}\right\}_{P \in \mathscr{b}_{i}}$. It should be mentioned that, in a suitable sense, $\mathbf{c}_{\text {dis }}$ is unitary on the imaginary axis.

(3) Denote by

$$
\mathscr{L}^{2}(G / \Gamma ; \mathscr{O} ; \mathscr{C})
$$

the Hilbert space of those measurable functions

$$
\mathbf{F}: \sqrt{-1} \check{\mathfrak{a}}_{\mathscr{C}} \rightarrow \mathscr{E}_{\text {dis }}(\mathscr{O} ; \mathscr{C})
$$

such that components are preserved and

$$
\mathbf{F}_{j}\left(\mathbf{w} \Lambda_{i}\right)=\mathbf{c}_{\operatorname{dis}}\left(\mathscr{C}_{j} \mid \mathscr{C}_{i}: \mathbf{w}: \Lambda_{i}\right) \mathbf{F}_{i}\left(\Lambda_{i}\right) \quad\left(\mathbf{w} \in W\left(\mathscr{C}_{j}, \mathscr{C}_{i}\right)\right)
$$

with inner product

$$
(\mathbf{F}, \mathbf{G})=\frac{1}{(2 \pi)^{l}} \frac{1}{*(\mathscr{C})} \sum_{k=1}^{r} \int_{\sqrt{-1} \check{\mathfrak{a}}_{\mathscr{q}_{k}}}\left(\mathbf{F}_{k}\left(\Lambda_{k}\right), \mathbf{G}_{k}\left(\Lambda_{k}\right)\right) \cdot\left|d \Lambda_{k}\right|,
$$

where $l=\operatorname{rank}(\mathscr{C}), r$ is the number of $G$-conjugacy classes in $\mathscr{C}$ and

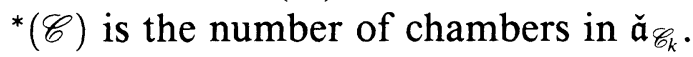

There is an isometric isomorphism

$$
\left\{\begin{array}{l}
\mathscr{L}^{2}(G / \Gamma ; \mathscr{O} ; \mathscr{C}) \rightarrow L^{2}(G / \Gamma ; \chi), \\
\mathbf{F} \mapsto \frac{1}{(2 \pi)^{l}} \frac{1}{*(\mathscr{C})} \int_{\sqrt{-1} \check{a}_{\mathscr{C}}} \mathbf{E}(\mathscr{C}: \mathbf{F}(\Lambda): \Lambda) \cdot|d \Lambda|,
\end{array}\right.
$$

whose image shall be denoted

$$
L^{2}(G / \Gamma ; \mathscr{O} ; \mathscr{C})
$$

Let $\left\{\varphi_{\mu}\right\}_{\mu}$ be an orthonormal basis for $\mathscr{E}_{\text {dis }}(\mathscr{O}, \mathscr{C})$ chosen such that each $\varphi_{\mu}$ lies in some $\mathscr{E}_{\text {dis }}(\delta, \mathscr{O}, \mathscr{C})$. The inverse isomorphism

$$
L^{2}(G / \Gamma ; \mathscr{O} ; \mathscr{C}) \rightarrow \mathscr{L}^{2}(G / \Gamma ; \mathscr{O} ; \mathscr{C})
$$


is given by

$$
f \mapsto \hat{f}=\sum_{\mu}\left\{\int_{G / \Gamma}\left(f(x), \mathbf{E}\left(\mathscr{C}: \varphi_{\mu}: \Lambda: x\right)\right) d x\right\} \varphi_{\mu} .
$$

This is the Eisenstein-Fourier transform of $f$.

There is an important connection with the principal series representations. The spectral decomposition of Langlands states:

$$
L^{2}(G / \Gamma ; \chi)=\sum_{\mathscr{C}} \sum_{\mathscr{O}} \bigoplus L^{2}(G / \Gamma ; \mathscr{O} ; \mathscr{C}),
$$

the spaces on the right being $L_{G / \Gamma}$-invariant. Denote by

$$
\operatorname{Ind}(G / \Gamma ; \mathscr{O} ; \mathscr{C}) \text {, }
$$

the direct integral

$$
\frac{1}{(2 \pi)^{l}} \int_{C(\mathscr{C})} \bigoplus \operatorname{Ind}_{\mathscr{C}}^{G}(\mathscr{O}, \Lambda) \cdot|d \Lambda|,
$$

which operates on the Hilbert space

$$
\mathscr{E}(G / \Gamma ; \mathscr{O} ; \mathscr{C})=\frac{1}{(2 \pi)^{l}} \int_{C(\mathscr{C})} \bigoplus \mathscr{E}_{\text {dis }}(\mathscr{O}, \Lambda) \cdot|d \Lambda|,
$$

where $C(\mathscr{C})$ is the positive chamber in $\sqrt{-1} \check{\mathfrak{a}}_{\mathscr{C}}$. There is a canonical identification

$$
\left\{\begin{array}{l}
\mathscr{L}^{2}(G / \Gamma ; \mathscr{O} ; \mathscr{C}) \rightarrow \mathscr{E}(G / \Gamma ; \mathscr{O} ; \mathscr{C}), \\
\left.\Phi \rightarrow \Phi\right|_{C(\mathscr{E})}
\end{array}\right.
$$

which, when composed with the Eisenstein-Fourier transform intertwines $L_{G / \Gamma}$ with Ind; viz.

$$
\left(L_{G / \Gamma}(\alpha) f\right)^{-}=\operatorname{Ind}(G / \Gamma ; \mathscr{O} ; \mathscr{C})(\alpha) \hat{f},
$$

for all $f \in L^{2}(G / \Gamma ; \mathscr{O} ; \mathscr{C})$ and $\alpha \in C_{c}^{\infty}(G)$.

(4) The upshot of the foregoing is that

$$
L_{\text {con }}^{2}(G / \Gamma ; \chi)=\sum_{\mathscr{C} \neq\{G\}} \sum_{\mathscr{O}} \bigoplus L^{2}(G / \Gamma ; \mathscr{O} ; \mathscr{C}) .
$$

Whence, for fixed $\mathscr{C} \neq\{G\}, L_{G / \Gamma}^{\text {con }}$ operates on

$$
L^{2}(G / \Gamma ; \mathscr{O} ; \mathscr{C})
$$

according to

$$
\frac{1}{(2 \pi)^{l}} \int_{C(\mathscr{C})} \bigoplus \operatorname{Ind}_{\mathscr{C}}^{G}(\mathscr{O}, \Lambda) \cdot|d \Lambda|
$$



then

Let $\alpha \in C_{c}^{\infty}(G ; K)$. Suppose that $f \in L^{2}(G / \Gamma ; \mathscr{O} ; \mathscr{C})(\mathscr{C} \neq\{G\})-$

$$
\begin{array}{rl}
\alpha * & f(x) \\
= & \frac{1}{(2 \pi)^{l}} \frac{1}{*(\mathscr{C})} \int_{\operatorname{Re}(\Lambda)=0} \alpha * \mathbf{E}(\mathscr{C}: \hat{f}(\Lambda): \Lambda: \cdot)(x)|d \Lambda| \\
= & \frac{1}{(2 \pi)^{l}} \frac{1}{*(\mathscr{C})} \sum_{\mu} \int_{\operatorname{Re}(\Lambda)=0} \mathbf{E}\left(\mathscr{C}: \operatorname{Ind}_{\mathscr{C}}^{G}(\mathscr{O}, \Lambda)(\alpha) \varphi_{\mu}: \Lambda: x\right) \\
& \times\left\{\int_{G / \Gamma}\left(f(y), \mathbf{E}\left(\mathscr{C}: \varphi_{\mu}: \Lambda: y\right)\right) d y\right\}|d \Lambda| .
\end{array}
$$

This computation motivates the following theorem.

Set

$$
\mathbf{C}_{\mu \nu}(\alpha: \mathscr{O}, \Lambda)=\left(\operatorname{Ind}_{\mathscr{C}}^{G}(\mathscr{O}, \Lambda)(\alpha) \varphi_{\nu}, \varphi_{\mu}\right)
$$

Form

$$
\begin{aligned}
& K_{\alpha}(x, y: \mathscr{O}, \Lambda) \\
& \quad=\sum_{\mu, \nu} \mathbf{C}_{\mu \nu}(\alpha: \mathscr{O}, \Lambda) \cdot \mathbf{E}\left(\mathscr{C}: \varphi_{\mu}: \Lambda: x\right) \cdot \mathbf{E}^{*}\left(\mathscr{C}: \varphi_{\nu}: \Lambda: y\right),
\end{aligned}
$$

where

$$
\begin{aligned}
\int_{G / \Gamma} & K_{\alpha}(x, y: \mathscr{O}, \Lambda) f(y) d y \\
= & \sum_{\mu, \nu} \mathbf{C}_{\mu \nu}(\alpha: \mathscr{O}, \Lambda) \cdot \mathbf{E}\left(\mathscr{C}: \varphi_{\mu}: \Lambda: x\right) \\
& \cdot \int_{G / \Gamma}\left(f(y), \mathbf{E}\left(\mathscr{C}: \varphi_{\nu}: \Lambda: y\right)\right) d y
\end{aligned}
$$

Write

$$
K_{\alpha}(x, y: \mathscr{O} ; \mathscr{C})
$$

in place of

$$
\frac{1}{(2 \pi)^{l}} \cdot \frac{1}{*(\mathscr{C})} \cdot \int_{\operatorname{Re}(\Lambda)=0} K_{\alpha}(x, y: \mathscr{O}, \Lambda) \cdot|d \Lambda|,
$$

and then put

$$
K_{\alpha}(x, y: \mathscr{C})=\sum_{\mathscr{O}} K_{\alpha}(x, y: \mathscr{O} ; \mathscr{C})
$$

Let $\mathscr{C}^{1}(G)$ denote Harish-Chandra's space of integrable rapidly decreasing functions. Let $\mathscr{C}^{1}(G ; K)$ denote the $K$-finite functions in $\mathscr{C}^{1}(G)$, with the $L F$-topology. 
TheOREM 1. Let $\alpha$ be element of $\mathscr{C}^{1}(G ; K)$-then $L_{G / \Gamma}^{\text {con }}(\alpha)$ is an integral operator on

$$
L_{\text {con }}^{2}(G / \Gamma ; \chi)
$$

with kernel

$$
K_{\alpha}^{\mathrm{con}}(x, y)=\sum_{\mathscr{C} \neq\{G\}} K_{\alpha}(x, y: \mathscr{C})
$$

continuous in each variable separately.

REMARKs. The form of $K_{\alpha}^{\text {con }}(x, y)$ follows directly from the preceding calculation. For the proof of the continuity, in slightly less generality, the reader is referred to $\S 8$ of [OW1].

2. The spectral decomposition for groups without Eisenstein series.

(1) Recall that the pair $(G, \Gamma)$ has been subject to a certain assumption. Let us make this assumption precise. Put

$$
\left\{\begin{array}{l}
Z=\text { analytic subgroup of } G \text { corresponding to the center of } \mathfrak{g}, \\
G_{c}=\text { analytic subgroup of } G \text { corresponding to the compact } \\
\text { ideals of } \mathfrak{g},
\end{array}\right.
$$

and

$$
\left\{\begin{array}{l}
\Gamma_{n}=\Gamma \cdot Z \cdot G_{c} / Z \cdot G_{c}, \\
G_{n}=G / Z \cdot G_{c} .
\end{array}\right.
$$

Define $E(G, \Gamma)$ to be the collection of split parabolic subgroups of $G$ obtained by pulling back to $G$ the percuspidal subgroups of $\Gamma_{n}$ in $G_{n}$ (cf. p. 37 of [OW1]).

Assumption. $E(G, \Gamma)$ comprises all $\Gamma$-percuspidal subgroups of $G$.

This assumption is entirely equivalent to the condition imposed by Langlands on page 16 of [L2] (cf. pp. 62-63 of [OW1]). It should be noted that an example of pair $(G, \Gamma)$ that does not satisfy this assumption is constructed on pp. 63-65 of [OW1].

(2) Henceforth we shall drop the assumption on $(G, \Gamma)$. It is not known whether a satisfactory theory of Eisenstein series exists for the pair $(G, \Gamma)$. However $\left(G_{n}, \Gamma_{n}\right)$ always possesses Eisenstein series. This fact is crucial for applications to the trace formula of the spectral decomposition that follows. 
Denote by $G^{0}$, the identity component of $G$. Set

$$
\left\{\begin{array}{l}
\Gamma^{0}=\Gamma \cap G^{0} \\
G_{n}^{0}=G^{0} / Z \cdot G_{c}, \\
\Gamma_{n}^{0}=\Gamma^{0} \cdot Z \cdot G_{c} / Z \cdot G_{c}, \\
\Gamma_{c}=\Gamma \cap Z \cdot G_{c}, \\
\Gamma_{Z}=\Gamma_{c} \cdot G_{c} \cap Z .
\end{array}\right.
$$

Observe that $G_{n}^{0}$ and $\Gamma_{n}^{0}$ may be viewed as subgroups of $G^{0}$ with the property that

Let

$$
\left\{\begin{array}{l}
G^{0}=Z \cdot G_{c} \cdot G_{n}^{0}, \\
Z \cdot G_{c} \cdot \Gamma^{0}=Z \cdot G_{c} \cdot \Gamma_{n}^{0} .
\end{array}\right.
$$

$$
I_{c}: L^{2}\left(G_{c} / G_{c} \cap \Gamma_{c} ; \chi\right) \rightarrow L^{2}\left(G_{c} \cdot \Gamma_{c} / \Gamma_{c} ; \chi\right)
$$

be the canonical isomorphism. Decompose

$$
L^{2}\left(G_{c} / G_{c} \cap \Gamma_{c} ; \chi\right)=\sum_{U_{c} \in \hat{G}_{c}} \bigoplus m_{U_{c}} U_{c} .
$$

Let $\chi_{c}$ be the left regular representation of $\Gamma_{Z}$ on $I_{c}\left(E\left(U_{c}\right)\right)$, where $E\left(U_{c}\right)$ is the representation space of $m_{U_{c}} U_{c}$. Since $G_{c} \cdot \Gamma_{c}=G_{c} \cdot \Gamma_{Z}$, it follows that

$$
\begin{aligned}
L^{2}\left(Z \cdot G_{c} / \Gamma_{c} ; \chi\right) & =\operatorname{Ind}_{\Gamma_{c} \cdot G_{c}}^{Z \cdot G_{c}}\left(L^{2}\left(G_{c} \cdot \Gamma_{c} / \Gamma_{c} ; \chi\right)\right) \\
& =\operatorname{Ind}_{\Gamma_{c} \cdot G_{c}}^{Z \cdot G_{c}}\left(\sum_{U_{c} \in \hat{G}_{c}} \bigoplus m_{U_{c}} U_{c} \otimes \chi_{c}\right) \\
& =\sum_{U_{c} \in \hat{G}_{c}} \bigoplus m_{U_{c}} U_{c} \otimes \operatorname{Ind}_{\Gamma_{Z}}^{Z}\left(\chi_{c}\right) .
\end{aligned}
$$

Put

$$
\tau=\operatorname{Ind}_{\Gamma_{Z}}^{Z}\left(\chi_{c}\right)
$$

Thus

$$
L^{2}\left(Z \cdot G_{c} / \Gamma_{c} ; \chi\right)=\sum_{U_{c} \in \hat{G}_{c}} \bigoplus m_{U_{c}} U_{c} \otimes \tau .
$$

The multiplicities $m_{U_{c}}$ shall be computed. By the Selberg trace formula for $L^{2}\left(G_{c} / G_{c} \cap \Gamma_{c} ; \chi\right)$,

$$
\begin{aligned}
& \sum_{U_{c} \in \hat{G}_{c}} m_{U_{c}} \operatorname{trace}\left(U_{c}(\alpha)\right) \quad\left(\alpha \in C^{\infty}\left(G_{c}\right)\right) \\
& =\sum_{\{\gamma\}} \operatorname{trace}(\chi(\gamma)) \cdot \operatorname{Vol}\left(\left(G_{c}\right)_{\gamma} /\left(G_{c} \cap \Gamma_{c}\right)_{\gamma}\right) \cdot \int_{G_{c} /\left(G_{c}\right)_{\gamma}} \alpha\left(x \gamma x^{-1}\right) d x,
\end{aligned}
$$


where

$$
\left\{\begin{array}{l}
\left(G_{c}\right)_{\gamma}=\text { the centralizer of } \gamma \text { in } G_{c}, \\
\left(G_{c} \cap \Gamma_{c}\right)_{\gamma}=\text { the centralizer of } \gamma \text { in } G_{c} \cap \Gamma_{c},
\end{array}\right.
$$

and $\sum_{\{\gamma\}}$ denotes the sum over the conjugacy classes in $G_{c} \cap \Gamma_{c}$. Insert $\alpha=\operatorname{trace}\left(\bar{U}_{c}\right)$ into the trace formula, to obtain

$$
m_{U_{c}}=\sum_{\{\gamma\}} \operatorname{trace}\left(\bar{U}_{c}(\gamma)\right) \cdot \operatorname{trace}(\chi(\gamma)) \cdot \operatorname{Vol}\left(G_{c} /\left(G_{c} \cap \Gamma\right)_{\gamma}\right) .
$$

(3) Let

$$
I_{0}: L^{2}\left(Z \cdot G_{c} / \Gamma_{c} ; \chi\right) \rightarrow L^{2}\left(Z \cdot G_{c} \cdot \Gamma^{0} / \Gamma^{0} ; \chi\right)
$$

be the canonical isomorphism. Let $\chi_{n}^{0}$ be the left regular representation of $\Gamma_{n}^{0}$ on $I_{0}\left(E\left(U_{c} \otimes \tau\right)\right)$, where $E\left(U_{c} \otimes \tau\right)$ is the representation space of $m_{U_{c}} U_{c} \otimes \tau$. Thence

$$
L^{2}\left(Z \cdot G_{c} \cdot \Gamma^{0} / \Gamma^{0} ; \chi\right)=\sum_{U_{c} \in \hat{G}_{c}} \bigoplus m_{U_{c}} U_{c} \otimes \tau \otimes \chi_{n}^{0} .
$$

Suppose that $\Gamma$ is contained in $G^{0}$-then

$$
\operatorname{Ind}_{\Gamma}^{G}(\chi)=\operatorname{Ind}_{G^{0}}^{G}\left(\operatorname{Ind}_{\Gamma}^{G^{0}}(\chi)\right) .
$$

Combining this observation with 2.1 yields

$$
L^{2}(G / \Gamma ; \chi)=\operatorname{Ind}_{G^{0}}^{G}\left\{\sum_{U_{c} \in \hat{G}_{c}} \bigoplus m_{U_{c}} U_{c} \otimes \tau \otimes \operatorname{Ind}_{\Gamma_{n}^{0}}^{G_{n}^{0}}\left(\chi_{n}^{0}\right)\right\} .
$$

Let $\alpha \in C_{c}^{\infty}(G)$. Put

$$
\begin{aligned}
& \alpha^{0}\left(U_{c}\right)(x) \\
& =\int_{G / G^{0}} \int_{Z} \int_{G_{c}} \alpha\left(w x y z w^{-1}\right) \operatorname{trace}(\tau(z)) \operatorname{trace}\left(U_{c}(y)\right) d w d z d y .
\end{aligned}
$$

Then

$$
\operatorname{trace}\left(L_{G / \Gamma}^{\mathrm{dis}}(\alpha)\right)=\sum_{U \in \hat{G}_{c}} m_{U_{c}} \operatorname{trace}\left(L_{G_{n}^{0} / \Gamma_{n}^{0}}^{\mathrm{dis}, \chi^{0}}\left(\alpha^{0}\left(U_{c}\right)\right),\right.
$$

where $L_{G_{n}^{0} / \Gamma_{n}^{0}}^{\text {dis } \chi^{0}}$ denotes the left regular representation on

$$
L_{\text {dis }}^{2}\left(G_{n}^{0} / \Gamma_{n}^{0} ; \chi_{n}^{0}\right)
$$


However, when $\Gamma$ is not contained in $G^{0}$ then

$$
L^{2}(G / \Gamma ; \chi)=\pi_{\Gamma^{0}}^{\Gamma}\left(L^{2}\left(G / \Gamma^{0} ; \chi\right)\right),
$$

where

$$
\pi_{\Gamma^{0}}^{\Gamma}(f)(x)=\frac{1}{\left[\Gamma: \Gamma^{0}\right]} \sum_{\gamma \in \Gamma / \Gamma^{0}} \chi(\gamma) f(x \gamma) .
$$

There does not seem to be any reasonable way to incorporate $\pi_{\Gamma^{0}}^{\Gamma}$ into the trace formula when $(G, \Gamma)$ does not possess Eisenstein series. In order to overcome this obstacle, an assumption shall be placed on $G$, which is satisfied by all connected groups. Assume that $G_{n}$ embeds in $G$ in such a way that

$$
Z G_{c} \cap G_{n}
$$

is discrete, and

$$
G=Z \cdot G_{c} \cdot G_{n} .
$$

More generally, assume that $(G, \Gamma)$ satisfies the following

$$
\left\{\begin{array}{l}
G=G_{1} \times G_{2} \\
\Gamma=\Gamma_{1} \times \Gamma_{2}
\end{array}\right.
$$

where $\Gamma_{2}$ is contained in $G_{2}^{0}$ and $G_{1}$ is a product of groups satisfying the assumption of $\S 2.1$ and groups $G^{\prime}$ for which $G_{n}^{\prime}$ embeds in $G^{\prime}$ as described above.

Let

$$
I: L^{2}\left(Z \cdot G_{c} / \Gamma_{c} ; \chi\right) \rightarrow L^{2}\left(Z \cdot G_{c} \cdot \Gamma / \Gamma ; \chi\right)
$$

be the canonical isomorphism. Let $\chi_{n}$ be the left regular representation of $\Gamma_{n}$ on $I\left(E\left(U_{c} \otimes \chi_{n}\right)\right)$-then

$$
L^{2}\left(Z \cdot G_{c} \cdot \Gamma / \Gamma ; \chi\right)=\sum_{U_{c} \in \hat{G}_{c}} \bigoplus m_{U_{c}} \tau \otimes U_{c} \otimes \chi_{n} .
$$

Ergo

$$
L^{2}(G / \Gamma ; \chi)=\operatorname{Ind}_{Z \cdot G_{c} \cdot \Gamma}^{G}\left(L^{2}\left(Z \cdot G_{c} \cdot \Gamma / \Gamma ; \chi\right)\right)
$$

(4) We shall now explicate the trace formula that arises from the decomposition (2.3), the situation in the case of (2.2) being entirely analogous.

Let $\alpha \in C_{c}^{\infty}(G)$. Put

$$
\alpha\left(U_{c}: x\right)=\int_{Z} \int_{G_{c}} \operatorname{trace}(\tau(z)) \cdot \operatorname{trace}\left(U_{c}(y)\right) \cdot \alpha(x y z) d z d y .
$$


Then $\alpha\left(U_{c}\right)$ belongs to $C_{c}^{\infty}\left(G_{n}\right)$, by the Schwarz kernel theorem (cf. Appendix 2.2 to Vol. I of [W1]). Let $L_{G_{n} / \Gamma_{n}}^{\text {dis, } \chi_{n}}$ denote the left regular representation of $G_{n}$ on

$$
L_{\text {dis }}^{2}\left(G_{n} / \Gamma_{n} ; \chi_{n}\right)
$$

Whence, on the assumption that $L_{G / \Gamma}^{\mathrm{dis}}(\alpha)$ is of the trace class,

$$
\begin{aligned}
& \operatorname{trace}\left(L_{G / \Gamma}^{\mathrm{dis}}(\alpha)\right) \\
& =\sum_{U_{c} \in \hat{G}_{c}} \bigoplus \sum_{\{\gamma\}} \operatorname{trace}\left(\bar{U}_{c}(\gamma)\right) \cdot \operatorname{trace}(\chi(\gamma)) \cdot \operatorname{Vol}\left(G_{c} /\left(G_{c} \cap \Gamma\right)_{\gamma}\right) \\
& \quad \cdot \operatorname{trace}\left(L_{G_{n} / \Gamma_{n}}^{\operatorname{dis}, \chi_{n}}\left(\alpha\left(U_{c}\right)\right)\right) .
\end{aligned}
$$

The function

$$
\alpha\left(U_{c}: x\right)
$$

can be further described by the Selberg trace formula for

$$
\tau=\operatorname{Ind}_{\Gamma_{Z}}^{Z}\left(\chi_{c}\right)
$$

Perhaps it is better to call it the Poisson summation formula since $Z$ is abelian. Indeed

$$
\alpha\left(U_{c}: x\right)
$$

equals

$$
\sum_{\delta \in \Gamma_{Z}} \operatorname{trace}\left(\chi_{c}(\delta)\right) \cdot \operatorname{Vol}\left(Z / \Gamma_{Z}\right) \cdot \int_{G_{c}} \operatorname{trace}\left(U_{c}(y)\right) \cdot \alpha(x y \delta) d y
$$

REMARK. Let $\alpha \in C_{c}^{\infty}(G ; K)$-then $\alpha\left(U_{c}\right)=0$ for all but finitely many $U_{c} \in \hat{G}_{c}$. Therefore, whenever $L_{G_{n} / \Gamma_{n}}^{\mathrm{dis}, \chi_{n}}(\alpha)$ is of the trace class, so is $L_{G / \Gamma}^{\text {dis, } \chi}(\alpha)$.

3. Truncating the kernels. In this section the basic properties of the truncation operator $Q^{\mathbf{H}}$ are reviewed. In addition, a partial truncation operator $Q_{N}$, due to Müller (cf. [MU1]), is introduced. The effect of truncating the kernels introduced in $\S 1$ is then investigated.

(1) Assume that $(G, \Gamma)$ satisfies the assumption of $\S 2.1$.

Let $\mathscr{C}(\Gamma)$ be the set of all $\Gamma$-cuspidal subgroups of $G$. Give $\mathfrak{a}_{\mathscr{C}(\Gamma)}$ the obvious definition. There is a natural order " $\leq$ " on $\mathfrak{a}_{\mathscr{C}(\Gamma)}$ that need not be specified until the applications. Let $\mathbf{H} \in \mathfrak{a}_{\mathscr{C}(\Gamma)}$ and $f \in$ $L_{\text {loc }}^{1}(G / \Gamma ; \chi)$-then the truncation operator $Q^{\mathbf{H}}$ is defined by

$$
Q^{\mathbf{H}} f(x)=\sum_{P \in \mathscr{C}(\Gamma)}(-1)^{\operatorname{rank}(P)} \chi_{P:]}\left(\mathbf{H}_{P}-H_{P}(x)\right) \cdot f^{P}(x),
$$


where $\chi_{P:]}$ is the characteristic function of the positive cone of $P$.

In order to state the salient properties of $Q^{\mathbf{H}}$, a few facts need to be recalled. Let $P$ be a fixed $\Gamma$-percuspidal subgroup of $G$. Let

$$
\mathfrak{S}_{t, \omega}=K \cdot A[t] \cdot \omega,
$$

where $\omega$ is a compact neighborhood of 1 in $M \cdot N$ and

$$
A[t]=\bigcap_{\alpha \in \Sigma_{P}^{0}}\left\{a \in A \mid \xi_{\alpha}(a) \leq t\right\}
$$

Here, $\Sigma_{P}^{0}$ is the set of simple roots determined by $P$. It follows from Lemma 2.11 of [OW1] that $t_{0}, \omega$, ${ }_{0} t$ and $s=\left\{\kappa_{i}: 1 \leq i \leq r\right\} \subset K$ can be chosen so that

$$
\left\{\begin{array}{l}
G=\mathfrak{S}_{t_{0}, \omega} \cdot \mathfrak{s} \cdot \Gamma, \\
\#\left\{\gamma \in \Gamma \mid \mathfrak{S}_{t_{0}, \omega} \cdot \mathfrak{s} \cdot \gamma \cap \mathfrak{S}_{t_{0}, \omega} \cdot \mathfrak{s} \neq \varnothing\right\}<\infty, \\
\mathfrak{S}_{t_{0}, \omega} \cdot \kappa_{i} \cdot \gamma \cap \mathfrak{S}_{0} t, \omega \cdot \kappa_{j}=\varnothing \quad(i \neq j), \\
\mathfrak{S}_{t_{0}, \omega} \cdot \kappa_{i} \cdot \gamma \cap \mathfrak{S}_{0, t, \omega} \cdot \kappa_{i} \neq \varnothing \Rightarrow \gamma \in \Gamma \cap P_{i},
\end{array}\right.
$$

where $P_{i}=\kappa_{i}^{-1} P \cdot \kappa_{i}$. In addition, we shall assume that $\left(\kappa_{i}^{-1} \omega \kappa_{i}\right)$. $\left(\Gamma \cap P_{i}\right)=M_{i} \cdot N_{i}$.

Put

$$
\Xi_{P}(x)=\inf _{\alpha \in \Sigma_{P}^{0}} \xi_{\alpha}(x) .
$$

Let $f \in L_{\text {loc }}^{1}(G / \Gamma ; \chi)$-then $f$ is said to be slowly increasing with exponent of growth $r(r \in \mathbf{R})$ if there is a constant $c>0$ such that

$$
\left|f\left(x \kappa_{i}\right)\right| \leq c \Xi_{P}^{r}(x) \quad\left(x \in \mathfrak{S}_{t_{0}, \omega}, 1 \leq i \leq r\right) .
$$

Let $S_{r}^{\infty}(G / \Gamma ; \chi)$ be comprised of all smooth $f \in L_{\text {loc }}^{1}(G / \Gamma ; \chi)$ such that for every right invariant differential operator $D, D f$ is slowly increasing with exponent of growth $r$-then the seminorms

$$
|f|_{r, D}=\sup _{1 \leq i \leq r} \sup _{x \in \mathfrak{S}_{t_{0}, \omega}} \Xi_{p}^{-r}(x)\left|D f\left(x \kappa_{i}\right)\right|
$$

endow $S_{r}^{\infty}(G / \Gamma ; \chi)$ with the structure of a Fréchet space. Denote by $R(G / \Gamma ; \chi)$, the space of functions on $G$ that are slowly increasing with exponent of growth $r$, for every real number $r$. The seminorms $|\cdot|_{r, 1}(r \in \mathbf{R})$ also provide a Fréchet space topology. The functions in $R(G / \Gamma ; \chi)$ are said to be rapidly decreasing.

Let us summarize the properties of the truncation operator that are of the most use. 
THEOREM 2.

(i) $\lim _{H \rightarrow-\infty} Q^{\mathbf{H}} f=f$ uniformly on compact subsets of $G$.

(ii) $Q^{\mathbf{H}}=I D$ on cusp forms.

(iii) $Q^{\mathbf{H}}: S_{r}^{\infty}(G / \Gamma ; \chi) \rightarrow R(G / \Gamma ; \chi)$ is continuous.

(iv) $Q^{\mathbf{H}}$ is a bounded linear operator on $L^{2}(G / \Gamma ; \chi)$.

In fact, there exists $\mathbf{H}_{0} \in \mathfrak{a}_{\mathscr{C}(\Gamma)}$ such that for all $\mathbf{H} \leq \mathbf{H}_{0}, Q^{\mathbf{H}}$ is an orthogonal projection on $L^{2}(G / \Gamma ; \chi)$ and as such

$$
\lim _{H \rightarrow-\infty} Q^{\mathbf{H}}=I D
$$

in the strong operator topology on $L^{2}(G / \Gamma ; \chi)$.

(2) Let $P^{*}$ be an element of $\mathscr{C}(\Gamma)$ different from $G$. Denote by $\mathscr{C}^{*}$ the association class containing $P^{*}$. Order the orbits $\mathscr{O}_{1}^{*}, \mathscr{O}_{2}^{*}, \ldots$ Choose an orthonormal basis $\left\{\varphi_{\mu}^{i}\right\}_{\mu=1}^{\infty}$ for $\mathscr{E}_{\text {dis }}\left(\mathscr{O}_{i}^{*} ; \mathscr{C}^{*}\right)$, such that each $\varphi_{\mu}^{i}$ lies in some $\mathscr{E}_{\text {dis }}\left(\delta, \mathscr{O}_{i}^{*} ; \mathscr{C}^{*}\right)$. Let $f \in L_{\text {loc }}^{1}(G / \Gamma ; \chi)$ and let $k \in K, m \in$ $M^{*}, a \in A^{*}$ and $n \in N^{*}$. Define the function

$$
\pi_{P^{*}, N}(f)(k m a n)
$$

to be

$$
\sum_{i=N+1}^{\infty} \sum_{\mu}\left\{\int_{K} \int_{M^{*} / \Gamma_{M^{*}}}\left(f^{P^{*}}\left(k^{*} m^{*} a\right), \varphi_{\mu, P^{*}}^{i}\left(k^{*} m^{*}\right)\right) d k^{*} d m^{*}\right\} \varphi_{\mu, P^{*}}^{i}(k m) \text {. }
$$

Set

$$
\pi_{G, N}(f)=f \text {. }
$$

The partial truncation operator $Q_{N}$ is defined by

$$
\left(Q_{N} f\right)(x)=\sum_{P^{*} \in \mathscr{C}(\Gamma)}(-1)^{\operatorname{rank}\left(P^{*}\right)} \chi_{\left.P^{*}:\right]}\left(\mathbf{H}_{0}-H_{P^{*}}(x)\right) \cdot \pi_{P^{*}, N}(f)(x),
$$

where $\mathbf{H}_{0}$ is a fixed element of $\mathfrak{a}_{\mathscr{C}(\Gamma)}$ that is sufficiently negative in a sense yet to be made precise.

(3) Specialize now to the case that $G$ is of $\Gamma$-rank 1 (i.e. the $\Gamma$ percuspidal subgroups of $G$ are of rank one). $\mathfrak{a}_{\mathscr{C}(\Gamma)}$ can be identified with $\prod_{i=1}^{r} \mathfrak{a}_{i}$. We shall restrict to the diagonal determined by $\mathfrak{s}$ and identify it with a. For $H \in \mathfrak{a}$, set $t_{H}=e^{\alpha(H)}\left(\Sigma_{P}^{0}=\{\alpha\}\right)$. Choose $H_{0} \in \mathfrak{a}$ such that $t_{H_{0}}<{ }_{0} t$-then it follows from (3.1) that for $t_{H}<_{0} t$ and $x \in \mathfrak{S}_{t_{0}, \omega} \cdot \mathfrak{s}$,

$$
Q^{H} f(x)= \begin{cases}f(x)-f^{P_{i}}(x): & x \in \mathfrak{S}_{t_{H}, \omega} \cdot \kappa_{i}(\exists i), \\ f(x): & x \notin \mathfrak{S}_{t_{H}, \omega} \cdot \mathfrak{s}\end{cases}
$$


and

$$
Q_{N} f(x)= \begin{cases}f(x)-\pi_{P_{l}, N}(f)(x): & x \in \mathfrak{S}_{t_{H_{0}}, \omega} \cdot \mathscr{H}_{i}(\exists i), \\ f(x): & x \notin \mathfrak{S}_{t_{H_{0}}, \omega} \cdot \mathfrak{s} .\end{cases}
$$

Using this formulation the following theorem is easily seen to be true.

Theorem 3.

(i) If $f \in L_{\mathrm{loc}}^{1}(G / \Gamma ; \chi)$ and $x \in \mathfrak{S}_{t_{0}, \omega} \cdot \mathfrak{s}$, then $Q^{H} \circ Q_{N} f(x)=Q_{N} \circ Q^{H} f(x)= \begin{cases}Q^{H} f(x): & x \in \mathfrak{S}_{t_{H}, \omega} \cdot \mathfrak{s}, \\ Q_{N} f(x): & x \notin \mathfrak{S}_{t_{H}, \omega} \cdot \mathfrak{s} .\end{cases}$

(ii) $\lim _{N \rightarrow \infty} Q_{N} f=f$ uniformly on compact subsets of $G$.

(iii) $Q_{N}=I D$ on cusp forms and on Eisenstein series associated with an automorphic form $\varphi_{\mu}^{i}$ for $i \leq N$.

(iv) $Q_{N}$ is an orthogonal projection on $L^{2}(G / \Gamma ; \chi)$ and as such

$$
\lim _{N \rightarrow \infty} Q_{N}=I D
$$

in the strong operator topology on $L^{2}(G / \Gamma ; \chi)$.

REMARK. I do not know whether Theorem 2 and Theorem 3 are valid without the assumption on $(G, \Gamma)$ specified in $\S 2$.

(4) Return now to the situation that the pair $(G, \Gamma)$ is of arbitrary rank.

Let $\alpha$ belong to $\mathscr{C}^{1}(G ; K)$. Define $\widetilde{K}_{\alpha}(x, y)$ to be any one of

$$
\left\{\begin{array}{l}
K_{\alpha}(x, y) \\
K_{\alpha}^{\text {dis }}(x, y) \\
K_{\alpha}^{\operatorname{con}}(x, y)
\end{array}\right.
$$

Let $Q_{1}^{H}, Q_{N}^{1}$ (resp., $Q_{2}^{H}, Q_{N}^{2}$ ) denote truncation in the first (resp., second) variable of $\widetilde{K}_{\alpha}(x, y)$. Lemma 8.1 of [OW1], combined with Theorems 1,2 and 3 imply that

$$
\left\{\begin{array}{l}
Q_{1}^{H} \widetilde{K}_{\alpha}(x, y), \\
Q_{2}^{H} \widetilde{K}_{\alpha}(x, y), \\
Q_{1}^{H} Q_{2}^{H} \widetilde{K}_{\alpha}(x, y)
\end{array}\right.
$$

are separately continuous (off a set of measure zero) and locally norm bounded on $G / \Gamma \times G / \Gamma$. Moreover, the functions

$$
\left\{\begin{array}{l}
\operatorname{trace}\left(Q_{1}^{H} \widetilde{K}_{\alpha}(x, x)\right), \\
\operatorname{trace}\left(Q_{2}^{H} \widetilde{K}_{\alpha}(x, x)\right), \\
\operatorname{trace}\left(Q_{1}^{H} Q_{2}^{H} \widetilde{K}_{\alpha}(x, x)\right)
\end{array}\right.
$$


are integrable on $G / \Gamma$. It follows from the Theorem in the appendix to $\S 8$ of [OW1] that

$$
Q^{H} \circ L_{G / \Gamma}\left(\alpha * \alpha^{*}\right) \circ Q^{H} \quad\left(\alpha^{*}(x)=\overline{\alpha\left(x^{-1}\right)}\right)
$$

is of the trace class. (Of course, the same is true when $L_{G / \Gamma}$ is replaced by $L_{G / \Gamma}^{\text {dis }}$ or $\left.L_{G / \Gamma^{\cdot}}^{\text {con }}\right)$ In fact,

$$
\operatorname{trace}\left(Q^{H} \circ L_{G / \Gamma}\left(\alpha * \alpha^{*}\right) \circ Q^{H}\right)=\int_{G / \Gamma} \operatorname{trace}\left(Q_{1}^{H} Q_{2}^{H} K_{\alpha * \alpha^{*}}(x, x)\right) d x .
$$

Thus

$$
Q^{H} \circ L_{G / \Gamma}(\alpha)
$$

is Hilbert-Schmidt (when $H$ is sufficiently negative). The theory of paramatrix (cf. Theorem 4.4 of [W2]) implies that for every integer $p \geq 1$, there exists an integer $N \geq 1$ and $\mu \in C_{c}^{p}(G), \nu \in C_{c}^{\infty}(G)$ such that

$$
\Delta^{N} \cdot \mu=\delta+\nu
$$

where $\delta$ is the dirac distribution at $1 \in G$ and $\Delta$ is the Laplacian on G. Ergo

$$
\alpha=\left(\Delta^{N} \cdot \alpha\right) * \mu-\alpha * \nu
$$

Thence

$$
Q^{H} \circ L_{G / \Gamma}(\alpha) \circ Q^{H} \quad\left(\alpha \in \mathscr{C}^{1}(G)\right)
$$

is of the trace class.

Observe

$$
\lim _{H^{\prime} \rightarrow-\infty} \int_{G / \Gamma}\left|Q_{1}^{H} Q_{2}^{H^{\prime}} \widetilde{K}_{\alpha}(x, x)\right| d x=\int_{G / \Gamma}\left|Q_{1}^{H} \widetilde{K}_{\alpha}(x, x)\right| d x,
$$

and if $H^{\prime} \leq H \ll 0$, then

$$
\begin{aligned}
\operatorname{trace}\left(Q^{H} \circ L_{G / \Gamma}(\alpha) \circ Q^{H^{\prime}}\right) & =\operatorname{trace}\left(Q^{H} \circ\left(Q^{H} \circ L_{G / \Gamma}(\alpha) \circ Q^{H^{\prime}}\right)\right) \\
& =\operatorname{trace}\left(\left(Q^{H} \circ L_{G / \Gamma}(\alpha) \circ Q^{H^{\prime}}\right) \circ Q^{H}\right) \\
& =\operatorname{trace}\left(Q^{H} \circ L_{G / \Gamma}(\alpha) \circ Q^{H}\right) .
\end{aligned}
$$

Therefore trace $\left(Q_{1}^{H} \widetilde{K}_{\alpha}(x, x)\right)$ and trace $\left(Q_{1}^{H} Q_{2}^{H} \widetilde{K}_{\alpha}(x, x)\right)$ have the same integral, which, by a similar argument, coincides with the integral of $\operatorname{trace}\left(Q_{2}^{H} \widetilde{K}_{\alpha}(x, x)\right)$. Proceeding in the same manner, it is easily seen that

$$
\operatorname{trace}\left(Q_{N} \circ L_{G / \Gamma}(\alpha) \circ Q_{N} \circ Q^{H}\right)
$$

is equal to the sum of

$$
\int_{G / \Gamma} \operatorname{trace}\left(Q_{2}^{H_{0}} K_{\alpha}(x, x)\right) d x \quad\left(H<H_{0}\right)
$$


and

$$
\int_{G / \Gamma} \operatorname{trace}\left(\left(Q_{2}^{H} \circ Q_{N}^{2}-Q_{2}^{H_{0}}\right) K_{\alpha}(x, x)\right) d x
$$

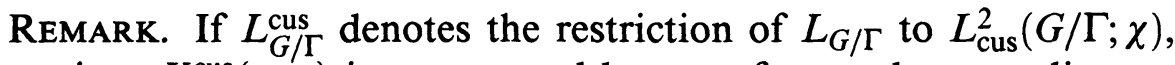
then since $K_{\alpha}^{\text {cus }}(x, y)$ is represented by cusp forms, the preceding results imply that $L_{G / \Gamma}^{\text {cus }}(\alpha)\left(\alpha \in \mathscr{C}^{1}(G)\right)$ is of the trace class.

Let $\alpha$ be an element of $\mathscr{C}^{1}(G ; K)$-then, in general, it is not known whether $L_{G / \Gamma}^{\text {dis }}(\alpha)$ is of the trace class (cf. [OW6] and [W3]). However, if $G$ is $\Gamma$-rank 1 , then Donnelly has answered this question in the affirmative. If $G$ is real rank 1 and $\delta \in \hat{K}$, then it follows from the spectral decomposition of Langlands that the $\delta$-isotypic component of

$$
L_{\text {res }}^{2}(G / \Gamma ; \chi)
$$

is finite dimensional. (Here the assumption of $\S 2$ is needed.) This observation combined with the remark supra implies the traceability of $L_{G / \Gamma}^{\text {dis }}(\alpha)$ directly. If $G$ is $\Gamma$-rank 0 , i.e. $\Gamma$ is cocompact in $G$, then

$$
L_{\text {cus }}^{2}(G / \Gamma ; \chi)=L^{2}(G / \Gamma ; \chi),
$$

so that $L_{G / \Gamma}(\alpha)\left(\alpha \in \mathscr{C}^{1}(G)\right)$ itself is of the trace class.

(5) For the remainder of the paper the pair $(G, \Gamma)$ shall be of $\Gamma$-rank 1 and satisfy the assumption of $\S 2.1$. Observe that there are only two $G$-conjugacy classes of $\Gamma$-cuspidal parabolic subgroups of $G$; viz., $\{G\}$ and $\mathscr{C}$.

Let $\alpha$ be an element of $C_{c}^{\infty}(G ; K)$. Then the results of Donnelly (cf. [D1]) imply that

is of the trace class. Since

$$
L_{G / \Gamma}^{\text {dis }}(\alpha)
$$

$$
Q_{N} \rightarrow I D
$$

in the strong operator topology on $L^{2}(G / \Gamma ; \chi)$, it follows immediately that

$$
\lim _{N \rightarrow \infty} \operatorname{trace}\left(Q_{N} \circ L_{G / \Gamma}(\alpha) \circ Q_{N}\right)=\operatorname{trace}\left(L_{G / \Gamma}(\alpha)\right) .
$$

Given a positive integer $N$, let

$$
L_{\text {con }}^{2, N}(G / \Gamma ; \chi)=\sum_{i=1}^{N} \bigoplus L^{2}\left(G / \Gamma ; \mathscr{O}_{i} ; \mathscr{C}\right),
$$

and let $L_{\text {dis }}^{2, N}(G / \Gamma ; \chi)$ be the complement of $L_{\text {con }}^{2, N}(G / \Gamma ; \chi)$ in

$$
Q_{N}\left(L^{2}(G / \Gamma ; \chi)\right)
$$


Denote by

$$
\left\{\begin{array}{l}
L_{G / \Gamma}^{\text {dis, } N} \\
L_{G / \Gamma}^{\text {con }, N}
\end{array}\right.
$$

the restriction of $Q_{N} \circ L_{G / \Gamma} \circ Q_{N}$ to

$$
\left\{\begin{array}{l}
L_{\mathrm{dis}}^{2, N}(G / \Gamma ; \chi) \\
L_{\mathrm{con}}^{2, N}(G / \Gamma ; \chi)
\end{array}\right.
$$

It is easily seen that

$$
L_{G / \Gamma}^{\mathrm{dis}, N}=Q_{N} \circ L_{G / \Gamma}^{\mathrm{dis}} \circ Q_{N}+T_{N}^{\mathrm{con}},
$$

where $T_{N}^{\text {con }}$ is the restriction of

$$
Q^{H_{0}} \circ L_{G / \Gamma}^{\text {con }} \circ Q^{H_{0}}
$$

to

$$
Q^{H_{0}}\left(\sum_{i=N+1}^{\infty} \bigoplus L^{2}\left(G / \Gamma ; \mathscr{O}_{i} ; \mathscr{C}\right)\right)
$$

Therefore $L_{G / \Gamma}^{\text {dis, } N}(\alpha)$ is of the trace class, with

$$
\operatorname{trace}\left(L_{G / \Gamma}^{\mathrm{dis}, N}(\alpha)\right)=\int_{G / \Gamma} \operatorname{trace}\left(K_{\alpha}^{\mathrm{dis}, N}(x, x)\right) d x,
$$

where

$$
K_{\alpha}^{\mathrm{dis}, N}(x, y)=Q_{N}^{1} Q_{N}^{2} K_{\alpha}(x, y)-\sum_{i=1}^{N} K_{\alpha}\left(x, y: \mathscr{O}_{i} ; \mathscr{C}\right),
$$

(cf. §1.4). Here, we have implicitly used (an obvious variant of) Theorem 2 on page 23 of [O1]. Furthermore,

$$
\lim _{N \rightarrow \infty} \operatorname{trace}\left(L_{G / \Gamma}^{\operatorname{dis}, N}(\alpha)\right)=\operatorname{trace}\left(L_{G / \Gamma}^{\text {dis }}(\alpha)\right) .
$$

4. The Selbert trace formula. In this section the pair $(G, \Gamma)$ shall satisfy the assumption of $\S 2.1$, and be of $\Gamma$-rank 1 .

On the basis of the work of Donnelly, the closed graph theorem implies

$$
\alpha \mapsto \operatorname{trace}\left(L_{G / \Gamma}^{\operatorname{dis}}(\alpha)\right)
$$

is continuous in the topology of $C_{c}^{\infty}(G ; K)$ (or even in the topology of $\left.\mathscr{C}^{1}(G ; K)\right)$. The remainder of this paper will be devoted to an explicit realization of this distribution. The techniques used are based on the work of Arthur, Müller, Osborne and Warner (cf. [A1], [MU1] and [OW2]). 
(1) Fix an element $\alpha$ of $C_{c}^{\infty}(G ; K)$-then

$$
\lim _{H \rightarrow-\infty} \operatorname{trace}\left(L_{G / \Gamma}^{\mathrm{dis}, N}(\alpha) \circ Q^{H}\right)=\operatorname{trace}\left(L_{G / \Gamma}^{\mathrm{dis}, N}(\alpha)\right)
$$

Write

$\operatorname{trace}\left(L_{G / \Gamma}^{\mathrm{dis}, N}(\alpha) \circ Q^{H}\right)=\int_{G / \Gamma} \operatorname{trace}\left(Q_{2}^{H} K_{\alpha}^{\mathrm{dis}, N}(x, x)\right) d x \quad\left(H<H_{0}\right)$

$$
\begin{aligned}
= & \int_{G / \Gamma} \operatorname{trace}\left(Q_{2}^{H_{0}} K_{\alpha}(x, x)\right) d x \\
& +\int_{G / \Gamma} \operatorname{trace}\left(\left(Q_{2}^{H} \circ Q_{N}^{2}-Q_{2}^{H_{0}}\right) K_{\alpha}(x, x)\right) d x \\
& -\sum_{i=1}^{N} \int_{G / \Gamma} \operatorname{trace}\left(Q_{2}^{H} K_{\alpha}\left(x, x: \mathscr{O}_{i} ; \mathscr{C}\right)\right) d x .
\end{aligned}
$$

The plan of attack is to send $H \rightarrow-\infty$ first and send $N \rightarrow \infty$ second.

We shall need the following fact from reduction theory. Let $C$ be a compact subset of $G$. Assume, without loss of generality, that

$$
C \subset \mathfrak{S}_{t_{0}, \omega}
$$

Parametrize $A$ by $\xi_{\alpha}(a(t))=t$. Let $\gamma \in \Gamma$-then

$$
a(t) \gamma a(-t) \in C \Rightarrow a(t) \gamma \in K \cdot A\left[t_{0}\right] \cdot a(t) M \cdot N \text {. }
$$

Thus, if $0<\varepsilon<{ }_{0} t$ is chosen small enough

$$
A\left[t_{0}\right] a(t) \subset A\left[{ }_{0} t\right] \quad(t<\varepsilon) .
$$

Hence, for all $0<t<\varepsilon$,

$$
a(t) \in \mathfrak{S}_{0 t, \omega} \cdot(\Gamma \cap P) \gamma^{-1} \cap \mathfrak{S}_{0} t, \omega \cdot(\Gamma \cap P),
$$

which, in view of (3.1), implies $\gamma \in \Gamma \cap P$. 
Let $x \in \mathfrak{S}_{t_{H_{0}}, \omega}\left(H_{0} \ll 0\right)$-then a consequence of the calculation supra is the following.

$$
\begin{aligned}
& \int_{N / N \cap \Gamma} \operatorname{pr}_{P} K_{\alpha}(x, x n) d n \\
& =\int_{N / N \cap \Gamma} \operatorname{pr}_{P}\left\{\sum_{\gamma \in \Gamma} \alpha\left(x \gamma n^{-1} x^{-1}\right) \chi(\gamma)\right\} d n \\
& =\int_{N / N \cap \Gamma} \operatorname{pr}_{P}\left\{\sum_{\gamma \in \Gamma / \Gamma \cap N} \sum_{\delta \in \Gamma \cap N} \alpha\left(x \gamma \delta^{-1} n^{-1} x^{-1}\right) \chi(\gamma) \chi\left(\delta^{-1}\right)\right\} d n \\
& =\int_{N}\left\{\sum_{\gamma \in \Gamma / \Gamma \cap N} \operatorname{pr}_{P} \alpha\left(x \gamma n x^{-1}\right) \chi(\gamma)\right\} d n \\
& =\int_{N}\left\{\sum_{\gamma \in \Gamma / \Gamma \cap P} \sum_{\delta \in \Gamma_{M}} \operatorname{pr}_{P} \alpha\left(x \gamma \delta n x^{-1}\right) \chi(\gamma) \chi(\delta)\right\} d n \\
& =\int_{N}\left\{\sum_{\gamma \in \Gamma \cap P / \Gamma \cap P} \sum_{\delta \in \Gamma_{M}} \operatorname{pr}_{P} \alpha\left(x \gamma \delta n x^{-1}\right) \chi(\gamma) \chi(\delta)\right\} d n \\
& =
\end{aligned}
$$

Observe that if $x \in \mathfrak{S}_{t_{0}, \omega} \cdot \mathfrak{s}$ and $f \in L_{\text {loc }}^{1}(G / \Gamma ; \chi)$,

$$
\begin{aligned}
&\left(Q^{H} \circ Q_{N}-Q^{H_{0}}\right)(f)(x) \\
& \quad=\left\{\begin{array}{ll}
f^{P_{\iota}}-\pi_{P_{i}, N}(f): t_{H}<\xi_{\alpha}\left(x \kappa_{i}^{-1}\right) \leq t_{H_{0}}(\exists i) \\
0: & \text { otherwise }
\end{array}\right\} .
\end{aligned}
$$

Moreover, if

$$
\alpha_{P}^{K}(m)=\int_{K} \int_{N} \alpha\left(k^{-1} m n k\right) d k d n,
$$

then the Schwarz kernel theorem implies that $\alpha_{P}^{K}$ belongs to $C_{c}^{\infty}(M)$. Whence

$$
K_{\alpha_{P}^{K}}\left(m_{1}, m_{2}\right)=\sum_{\delta \in \Gamma_{M}} \operatorname{pr}_{P}\left\{\alpha_{P}^{K}\left(m_{1} \delta m_{2}^{-1}\right) \chi(\delta)\right\}
$$

is the integral kernel of the trace class operator $L_{M / \Gamma_{M}}\left(\alpha_{P}^{K}\right)$. (Recall that $M / \Gamma_{M}$ is compact.) An elementary calculation now shows that

$$
\int_{G / \Gamma} \operatorname{trace}\left(\left(Q_{2}^{H} \circ Q_{N}^{2}-Q_{2}^{H_{0}}\right) K_{\alpha}(x, x)\right) d x
$$


is equal to

$$
\frac{\alpha\left(H_{0}\right)-\alpha(H)}{\|\alpha\|} \cdot \operatorname{trace}\left(L_{M / \Gamma_{M}}\left(\alpha_{P}^{K}\right) \cdot \tau_{N}\right)
$$

where

$$
\tau_{N}: \sum_{i=1}^{\infty} \bigoplus \mathscr{E}_{\text {dis }}\left(\mathscr{O}_{i} ; \mathscr{C}\right) \rightarrow \sum_{i=1}^{N} \bigoplus_{\text {dis }}\left(\mathscr{O}_{i} ; \mathscr{C}\right)
$$

is the orthogonal projection. The notation is poor because $\alpha$ is being used to denote both a simple root and a function. There should be no ambiguity.

(2) Let

$$
\mathbf{c}(\Lambda)=\mathbf{c}_{\text {dis }}(\mathscr{C} \mid \mathscr{C}:-\mathbf{1}: \Lambda),
$$

where -1 is the unique nonidentity element of $\mathbf{W}(\mathscr{C}, \mathscr{C})$. As a function on

$$
\mathscr{E}_{\text {dis }}(\delta, \mathscr{O} ; \mathscr{C}) \text {, }
$$

$\mathrm{c}(\Lambda)$ is unitary on the imaginary axis and

$$
\mathbf{c}(\Lambda)^{*}=\mathbf{c}(\bar{\Lambda}) \text {. }
$$

The following functional equations are satisfied

$$
\left\{\begin{array}{l}
\mathbf{c}(\Lambda) \mathbf{c}(-\Lambda)=I D, \\
\mathbf{E}(\mathscr{C}: \mathbf{c}(\Lambda) \varphi:-\Lambda)=\mathbf{E}(\mathscr{C}: \varphi: \Lambda)
\end{array}\right.
$$

In view of the identifications, write

$$
\pi_{\Lambda}(\alpha)=\operatorname{Ind}_{\mathscr{C}}^{G}\left(\mathscr{O}_{i}, \Lambda\right)(\alpha) \quad(\Lambda \in \check{\mathfrak{a}} \otimes \mathbf{C}) .
$$

It follows that

$$
K_{\alpha}\left(x, y: \mathscr{O}_{i}, \Lambda\right)=\sum_{\mu} \mathbf{E}\left(\mathscr{C}: \pi_{\Lambda}(\alpha) \varphi_{\mu}^{i}: \Lambda: x\right) \cdot \mathbf{E}^{*}\left(\mathscr{C}: \varphi_{\mu}^{i}: \Lambda: y\right) .
$$

Let $\Lambda \in \sqrt{-1} \check{\mathfrak{a}}$ and $\zeta \in \mathfrak{a}$ with $\Lambda \neq 0$-then the $L^{2}$ inner product formula of Langlands (cf. p. 135 of [L2] and [R2]) shows that

$$
\left(Q^{H} \mathbf{E}\left(\mathscr{C}: \pi_{\Lambda}(\alpha) \varphi: \Lambda+\zeta\right), Q^{H} \mathbf{E}(\mathscr{C}: \varphi: \Lambda+\zeta)\right)_{G / \Gamma}
$$

is equal to

$$
\begin{array}{r}
\frac{1}{2 \zeta\left(H_{\alpha}\right)}\left\{\mathrm{e}^{-2 \zeta(H)}\left(\mathbf{c}(\Lambda+\zeta) \pi_{\Lambda}(\alpha) \varphi, \mathbf{c}(\Lambda+\zeta) \varphi\right)-\mathrm{e}^{2 \zeta(H)}\left(\pi_{\Lambda}(\alpha) \varphi, \varphi\right)\right\} \\
+\frac{1}{2 \Lambda\left(H_{\alpha}\right)}\left\{\mathrm{e}^{-2 \Lambda(H)}\left(\mathbf{c}(\Lambda+\zeta) \pi_{\Lambda}(\alpha) \varphi, \varphi\right)\right. \\
\left.-e^{2 \Lambda(H)}\left(\pi_{\Lambda}(\alpha) \varphi, \mathbf{c}(\Lambda+\zeta) \varphi\right)\right\}
\end{array}
$$


where $\alpha\left(H_{\alpha}\right)=\|\alpha\|$. Letting $\zeta \rightarrow 0$, obtains

$$
\begin{aligned}
& -\frac{2 \alpha(H)}{\|\alpha\|}\left(\pi_{\Lambda}(\alpha) \varphi, \varphi\right)+\left(\mathbf{c}^{\prime}(\Lambda) \pi_{\Lambda}(\alpha) \varphi, \mathbf{c}(\Lambda) \varphi\right) \\
& \quad+\frac{1}{2 \Lambda\left(H_{\alpha}\right)}\left\{e^{-2 \Lambda(H)}\left(\mathbf{c}(\Lambda) \pi_{\Lambda}(\alpha) \varphi, \varphi\right)-e^{2 \Lambda(H)}\left(\pi_{\Lambda}(\alpha) \varphi, \mathbf{c}(\Lambda) \varphi\right)\right\}
\end{aligned}
$$

Therefore

$$
\begin{gathered}
\operatorname{trace}\left(L_{G / \Gamma}^{\operatorname{con}, N}(\alpha) \circ Q^{H}\right)=\sum_{i=1}^{N} \int_{G / \Gamma} \operatorname{trace}\left(Q_{2}^{H} K_{\alpha}(x, x: \mathscr{O} ; ; \mathscr{C})\right) d x \\
=\frac{1}{4 \pi} \cdot \sum_{i=1}^{N} \int_{\operatorname{Re}(\Lambda)=0} \int_{G / \Gamma} \operatorname{trace}\left(Q_{2}^{H} K_{\alpha}\left(x, x: \mathscr{O}_{i}, \Lambda\right)\right) \cdot|d \Lambda| d x \\
=\frac{1}{4 \pi} \cdot \sum_{i=1}^{N} \sum_{\mu} \int_{\operatorname{Re}(\Lambda)=0}\left(Q^{H} \mathbf{E}\left(\mathscr{C}: \pi_{\Lambda}(\alpha) \varphi_{\mu}: \Lambda\right),\right. \\
\left.Q^{H} \mathbf{E}\left(\mathscr{C}: \varphi_{\mu}: \Lambda\right)\right)_{G / \Gamma} \cdot|d \Lambda|
\end{gathered}
$$

which is equal to $1 / 4 \pi$ times the sum over $\sum_{i=1}^{N}$ of the integral over $\operatorname{Re}(\Lambda)=0$ of the sum of the following four terms:

$$
\begin{gathered}
-2 \frac{\alpha(H)}{\|\alpha\|} \operatorname{trace}\left(\operatorname{Ind}_{\mathscr{C}}^{G}\left(\mathscr{O}_{i}, \Lambda\right)(\alpha)\right), \\
\operatorname{trace}\left(\operatorname{Ind}_{\mathscr{C}}^{G}\left(\mathscr{O}_{i}, \Lambda\right)(\alpha) \cdot \mathbf{c}(\bar{\Lambda}) \cdot \mathbf{c}^{\prime}(\Lambda)\right), \\
\frac{1}{2 \Lambda\left(H_{\alpha}\right)} e^{-2 \Lambda(H)} \operatorname{trace}\left(\operatorname{Ind}_{\mathscr{C}}^{G}\left(\mathscr{O}_{i}, \Lambda\right)(\alpha) \cdot \mathbf{c}(\Lambda)\right),
\end{gathered}
$$

and

$$
-\frac{1}{2 \Lambda\left(H_{\alpha}\right)} e^{2 \Lambda(H)} \operatorname{trace}\left(\operatorname{Ind}_{\mathscr{C}}^{G}\left(\mathscr{O}_{i}, \Lambda\right)(\alpha) \cdot \mathbf{c}(\bar{\Lambda})\right) .
$$

Consider the term (4.5). It is readily computed that

$$
\operatorname{trace}\left(\operatorname{Ind}_{\mathscr{C}}^{G}\left(\mathscr{O}_{i}, \Lambda\right)(\alpha)\right)
$$

is given by

$$
\int_{A} \int_{M / \Gamma_{M}} \operatorname{trace}\left\{\sum_{\delta \in \Gamma_{M}} \operatorname{pr}_{P} \alpha_{P}^{K}\left(m \delta m^{-1} a\right) \chi(\delta)\right\} \xi_{-(\Lambda-\rho)} d a d m
$$

The Schwartz kernel theorem implies that

$$
a \mapsto \operatorname{trace}\left(L_{M / \Gamma_{M}}\left(\alpha_{P}^{K}(\cdot: a)\right)\right)
$$


belongs to $C_{c}^{\infty}(A)$; thus

$$
\operatorname{trace}\left(\operatorname{Ind}_{\mathscr{C}}^{G}\left(\mathscr{O}_{i}, \Lambda\right)(\alpha)\right)
$$

is rapidly decreasing. Moreover

$$
\sum_{i=1}^{N} \frac{1}{4 \pi} \int_{\operatorname{Re}(\Lambda)=0} \operatorname{trace}\left(\text { Ind }_{\mathscr{C}}^{G}\left(\mathscr{O}_{i}, \Lambda\right)(\alpha)\right)|d \Lambda|
$$

is equal to

$$
\frac{1}{2} \operatorname{trace}\left(L_{M / \Gamma_{M}}\left(\alpha_{P}^{K}\right) \cdot \tau_{N}\right),
$$

by Fourier inversion. Hence the contribution of terms of the form (4.5) to (4.3) cancels the part of (4.4) depending on $H$.

Consider the terms (4.7) and (4.8). Parametrize $\sqrt{-1}$ ă and $\mathfrak{a}$ by $\Lambda=\sqrt{-1} \zeta \alpha /\|\alpha\|$ and $2 H=-\xi H_{\alpha}-$ then $\Lambda\left(H_{\alpha}\right)=\sqrt{-1} \zeta$. Write

$$
\int_{\operatorname{Re}(\Lambda)=0}(4.7)+(4.8)|d \Lambda|
$$

as the sum of

$$
\int_{|\zeta|>\varepsilon}(4.7) d \zeta+\int_{|\zeta|>\varepsilon}(4.8) d \zeta
$$

and

$$
\int_{-\varepsilon}^{\varepsilon}(4.7)+(4.8) d \zeta
$$

The Riemann-Lebesgue lemma implies that both integrals in (4.9) are $o(H)$. Express (4.10) as the sum of

$$
\int_{-\varepsilon}^{\varepsilon} \cos (\xi \zeta) \cdot \operatorname{trace}\left(\operatorname{Ind}_{\mathscr{C}}^{G}\left(\mathscr{O}_{i}, \zeta\right)(\alpha) \cdot\left(\frac{\mathbf{c}(\zeta)-\mathbf{c}(-\zeta)}{2 \sqrt{-1} \zeta}\right)\right) d \zeta
$$

and

$$
\int_{-\varepsilon}^{\varepsilon} \frac{\sin (\xi \zeta)}{\zeta} \cdot \operatorname{trace}\left(\operatorname{Ind}_{\mathscr{C}}^{G}\left(\mathscr{O}_{i}, \zeta\right)(\alpha) \cdot\left(\frac{\mathbf{c}(\zeta)+\mathbf{c}(-\zeta)}{2}\right)\right) d \zeta
$$

Another application of the Riemann-Lebesgue lemma shows that (4.11) is $o(H)$. On the other hand, suppose $g \in L^{1}(\mathbf{R})$ is differentiable at $0-$ then by writing

$$
g(\zeta)=g(0)+\zeta\left\{\frac{g(\zeta)-g(0)}{\zeta}\right\}
$$

it follows that

$$
\lim _{\xi \rightarrow \infty} \int_{-\varepsilon}^{\varepsilon} \frac{\sin (\xi \zeta)}{\zeta} g(\zeta) d \zeta=\pi g(0)
$$


Then the limit as $H \rightarrow-\infty$ of $(4.12)$ is

$$
\pi \operatorname{trace}\left(\operatorname{Ind}_{\mathscr{C}}^{G}\left(\mathscr{O}_{i}, 0\right)(\alpha) \cdot \mathbf{c}(0)\right) .
$$

(3) Let us summarize what has been shown so far.

$$
\operatorname{trace}\left(L_{G / \Gamma}^{\mathrm{dis}, N}(\alpha) \circ Q^{H}\right) \quad \bmod (o(H))
$$

is equal to the sum of

$$
\begin{gathered}
\int_{G / \Gamma} \operatorname{trace}\left(Q_{2}^{H_{0}} K_{\alpha}(x, x)\right) d x, \\
\frac{\alpha\left(H_{0}\right)}{\|\alpha\|} \cdot \operatorname{trace}\left(L_{M / \Gamma_{M}}\left(\alpha_{P}^{K}\right) \cdot \tau_{N}\right), \\
-\frac{1}{4 \pi} \cdot \sum_{i=1}^{N} \int_{\operatorname{Re}(\Lambda)=0} \operatorname{trace}\left(\operatorname{Ind}_{\mathscr{C}}^{G}\left(\mathscr{O}_{i}, \Lambda\right)(\alpha) \cdot \mathbf{c}(\bar{\Lambda}) \cdot \mathbf{c}^{\prime}(\Lambda)\right) \cdot|d \Lambda|,
\end{gathered}
$$

and

$$
-\frac{1}{4} \cdot \sum_{i=1}^{N} \operatorname{trace}\left(\operatorname{Ind}_{\mathscr{C}}^{G}\left(\mathscr{O}_{i}, 0\right)(\alpha) \cdot \mathbf{c}(0)\right) .
$$

Send $H \rightarrow-\infty$-then send $N \rightarrow \infty$. Hence

$$
\operatorname{trace}\left(L_{G / \Gamma}^{\mathrm{dis}}(\alpha)\right)
$$

is equal to the sum of

$$
\begin{gathered}
\int_{G / \Gamma} \operatorname{trace}\left(Q_{2}^{H_{0}} K_{\alpha}(x, x)\right) d x, \\
\frac{\alpha\left(H_{0}\right)}{\|\alpha\|} \cdot \operatorname{trace}\left(L_{M / \Gamma_{M}}\left(\alpha_{P}^{K}\right)\right), \\
-\frac{1}{4 \pi} \cdot \sum_{\mathscr{O}} \int_{\operatorname{Re}(\Lambda)=0} \operatorname{trace}\left(\operatorname{Ind}_{\mathscr{C}}^{G}(\mathscr{O}, \Lambda)(\alpha) \cdot \mathbf{c}(\bar{\Lambda}) \cdot \mathbf{c}^{\prime}(\Lambda)\right) \cdot|d \Lambda|,
\end{gathered}
$$

and

$$
-\frac{1}{4} \sum_{\mathscr{O}} \operatorname{trace}\left(\operatorname{Ind}_{\mathscr{C}}^{G}(\mathscr{O}, 0)(\alpha) \cdot \mathbf{c}(0)\right) .
$$

Observe that $\mathbf{c}(0)$ extends to a bounded operator on

$$
\sum_{\mathscr{O}} \bigoplus \mathscr{E}_{\mathrm{dis}}(\mathscr{O} ; \mathscr{C}) \text {. }
$$

Let $\hat{\alpha}_{P}^{K}(m: \Lambda)$ denote the Fourier transform of the function

$$
a \mapsto \alpha_{P}^{K}(m a) .
$$


Then (4.16) is quickly seen to equal

$$
-\frac{1}{4} \operatorname{trace}\left(L_{M / \Gamma_{M}}\left(\hat{\alpha}_{P}^{K}(\cdot: 0)\right) \cdot \mathbf{c}(0)\right) \text {. }
$$

Whence, Weierstrass' theorem on conditional convergence implies that the sum in (4.15) converges absolutely.

(4) Denote by $T_{H_{0}}(\alpha)$ the sum of (4.13) and (4.14)-then $T_{H_{0}}$ and (4.16) extend to distributions on $C_{c}^{\infty}(G)$. Thus (4.15) must be a distribution on $C_{c}^{\infty}(G ; K)$. If it could be shown that there are constants $C$ and $L(C>0)$, independent of $\delta$ and $\mathscr{O}$, such that

$$
\left\|\mathbf{c}^{\prime}(\Lambda)\right\|_{O P} \leq C(1+\|\delta\|+\|\mathscr{O}\|+\|\Lambda\|)^{L} \quad(\Lambda \in \sqrt{-1} \mathfrak{a}),
$$

then the integral series in (4.15) is absolutely convergent and (4.15) extends to a distribution on $C_{c}^{\infty}(G)$. Here

$$
\left\{\begin{array}{l}
\|\delta\|=\left|\left\langle\delta, \omega_{K}\right\rangle\right| \\
\|\mathscr{O}\|=|\langle\mathscr{O}, \omega\rangle|
\end{array}\right.
$$

where

$$
\left\{\begin{array}{l}
\omega_{K}=\text { the Casimir of } K, \\
\omega=\text { the Casimir of } G,
\end{array}\right.
$$

and $\|\cdot\|_{O P}$ is the operator norm on

$$
\mathscr{E}_{\text {dis }}(\delta, \mathscr{O} ; \mathscr{C}) \text {. }
$$

This, of course, would imply that

$$
\alpha \mapsto L_{G / \Gamma}^{\mathrm{dis}}(\alpha)
$$

is also a distribution on $C_{c}^{\infty}(G)$ (or even on $\mathscr{C}^{1}(G)$ ). In particular, $L_{G / \Gamma}^{\text {dis }}(\alpha)$ is of the trace class for all $\alpha$ in $C_{c}^{\infty}(G)$.

The term $T_{H_{0}}(\alpha)$ is now unraveled mod $o\left(H_{0}\right)$ into orbital integrals corresponding to the semisimple elements of $\Gamma$ and a term

$$
\lim _{s \rightarrow 0}\left(s \mathbf{\Psi}_{\alpha}(\delta: s)\right),
$$

corresponding to the non-semisimple elements of $\Gamma$. This is done by Osborne and Warner in [OW2], pp. 56-92. In particular see the formula on page 93 of [OW2] for the complete trace formula. Just recently, the non-semisimple term (4.17) has been completely explicated by Hoffman (cf. [H1]), in terms of zeta functions attached to prehomogeneous vector spaces. The argument is quite analogous to the R-rank 1 situation (cf. [W2]). 
Added in proof. By utilizing a result of Arthur [cf. Theorem 8.1; Amer. J. Math., Vol. 104, No. 6, pp. 1289-1336], it can be shown that the integral series in (4.15) is absolutely convergent and hence each of its terms are distributions on $C_{c}^{\infty}(G ; K)$.

\section{REFERENCES}

[A1] James Arthur, The Selberg trace formula for groups of F-rank one, Annals of Math., 100, No. 2 (1974), 326-385.

[A2] $\quad$ Trace formula for reductive groups I: Terms associated to classes in $G(\mathbf{Q})$, Duke Math. J., 45 (1978), 911-952.

[A3] Eisenstein series and the trace formula, Proc. Symposia in Pure Math., 33, Part I, (1979), 253-274.

[A4] _ A trace formula for reductive groups II: Applications of a truncation operator, Compositio Math., 40, Fasc. 1 (1980), 87-121.

[A5] _ On the inner product of truncated Eisenstein series, Duke Math. J., 49, No. 1 (1982), 35-70.

[D1] Harold Donnelly, Eigenvalue estimates for certain noncompact manifolds, Michigan Math. J., 31 (1984), 349-357.

[HC1] Harish-Chandra, Automorphic Forms on Semisimple Lie Groups, Lecture Notes in Math. 62, Springer-Verlag, 1968.

[H1] Werner Hoffman, The non-semisimple term in the trace formula for rank one lattices, preprint.

[L1] Robert P. Langlands, Eisenstein series, Proc. Symposia in Pure Math., 9 (1966), 235-252.

[L2] _ On the Functional Equations Satisfied by Eisenstein Series, Lecture Notes in Math. 544, Springer-Verlag, 1976.

[L3] Dimension of space of automorphic forms, Proc. Symposia in Pure Math., 9 (1966), 253-257.

[M1] Polly Moore, Generalized Eisenstein series: Incorporation of a nontrivial representation of $\Gamma$, Thesis, University of Washington, 1979.

[MU1] Werner Müller, On the Selberg trace formula for rank one lattices, preprint. [O1] M. Scott Osborne, Spectral Theory and Uniform Lattices, Lecture Notes in Representation Theory, Univ. of Chicago Press, Chicago, Illinois, 1977.

[OW1] M. Scott Osborne and Garth Warner, The Theory of Eisenstein Systems, Academic Press, 1981.

[OW2] _ The Selberg trace formula I: $\Gamma$-rank one lattices, Crelle's J., 324 (1981), 1-113.

[OW3] $\quad$, The Selberg trace formula II: Partition, reduction, truncation, Pacific J. Math., 106 (1983), 307-496.

[OW4] - The Selberg trace formula III: Inner product formula (initial considerations), Memoirs of the Amer. Math. Soc., 283, June 1983.

[OW5] _ The Selberg Trace Formula IV: Inner Product Formulae (Final Consideration), Lecture Notes in Math., 1024, Springer-Verlag, 1983.

[OW6] - The Selberg trace formula V: questions of trace class, Trans. Amer. Math. Soc., 286 (1984), 351-376.

[R1] Paul F. Ringseth, Invariant Eisenstein systems, Ph. D. dissertation, University of Washington, June 1986.

[R2] - On the analytic continuation of $\mathbf{c}$-functions, preprint. 
[S1] Atle Selberg, Harmonic analysis and discontinuous groups in weakly symmetric Riemannian spaces with applications to Dirichlet series, J. Indian Math. Soc., 20 (1956), 47-87.

[S2] _ Discontinuous Groups and Harmonic Analysis, Proc. Int. Congress of Math., Stockholm, 1962.

[V1] A. B. Venkov, Spectral Theory of Automorphic Functions, Proc. of the Steklov Institute of Mathematics, 153 (1981).

[W1] G. Warner, Harmonic Analysis on Semi-Simple Lie Groups, Vols. I and II, Springer-Verlag, 1972.

[W2] - Selberg's trace formula for nonuniform lattices: The R-rank one case, Advances in Math. Studies, 6 (1979), 1-142.

[W3] _ Traceability on the discrete spectrum, Contemporary Math., 53 (1986).

[W4] Elementary aspects of the theory of Hecke operators, to appear.

Received December 10, 1986.

UNIVERSITY OF MiCHIGAN

ANN ARBOR, MI 48109 


\section{PACIFIC JOURNAL OF MATHEMATICS}

\section{EDITORS}

V. S. VARADARAJAN

(Managing Editor)

University of California

Los Angeles, CA 90024

Herbert Clemens

University of Utah

Salt Lake City, UT 84112

R. FINN

Stanford University

Stanford, CA 94305
HERMANN FLASCHKA

University of Arizona

Tucson, AZ 85721

RAMESh A. GANGOLLI

University of Washington Seattle, WA 98195

VAUGHAN F. R. JONES

University of California

Berkeley, CA 94720
ROBION KIRBY

University of California

Berkeley, CA 94720

C. C. MOORE

University of California

Berkeley, CA 94720

HAROLD STARK

University of California, San Diego

La Jolla, CA 92093

\section{ASSOCIATE EDITORS}
R. AREnS
E. F. BECKENBACH
B. H. NEUMANN
F. WOLF
K. YOSHIDA (1906-1982)

\section{SUPPORTING INSTITUTIONS}

UNIVERSITY OF ARIZONA

UNIVERSITY OF OREGON

UNIVERSITY OF BRITISH COLUMBIA UNIVERSITY OF SOUTHERN CALIFORNIA

CALIFORNIA INSTITUTE OF TECHNOLOGY

UNIVERSITY OF CALIFORNIA

STANFORD UNIVERSITY

MONTANA STATE UNIVERSITY

UNIVERSITY OF HAWAII

UNIVERSITY OF NEVADA, RENO

UNIVERSITY OF TOKYO

NEW MEXICO STATE UNIVERSITY

UNIVERSITY OF UTAH

OREGON STATE UNIVERSITY

WASHINGTON STATE UNIVERSITY

UNIVERSITY OF WASHINGTON 


\section{Pacific Journal of Mathematics}

\section{Vol. 133, No. $1 \quad$ March, 1988}

John Anderson, Finitely generated algebras and algebras of solutions to partial differential equations $\ldots \ldots \ldots \ldots \ldots \ldots \ldots \ldots \ldots \ldots \ldots \ldots \ldots$

Junichi Aramaki, On an extension of the Ikehara Tauberian theorem . . . . . 13

Giacomo Monti Bragadin, Abstract Riemannian stratifications .......... 31

Lawrence James Brenton and Richard Hill, On the Diophantine equation

$1=\sum 1 / n_{i}+1 / \prod n_{i}$ and a class of homologically trivial complex

surface singularities .................................41

C. Bruce Hughes, Controlled homotopy topological structures $\ldots . \ldots \ldots \ldots 69$

Peter Wilcox Jones and Takafumi Murai, Positive analytic capacity but zero Buffon needle probability . ...........................999

Gary M. Lieberman, Hölder continuity of the gradient at a corner for the

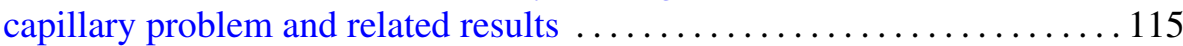

Feng Luo, Representing homology classes of $C \mathbf{P}^{2} \overline{C \mathbf{P}^{2}}$

Claudio Nebbia, Groups of isometries of a tree and the Kunze-Stein

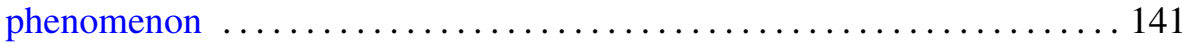

Stefan Richter, Unitary equivalence of invariant subspaces of Bergman and

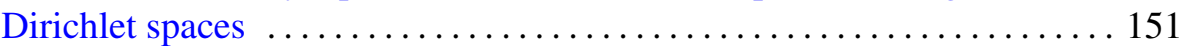

Paul Frederick Ringseth, The Selberg trace formula for groups without Eisenstein series ..................................... 157

Abderrazzak Sersouri, The Mazur property for compact sets $\ldots \ldots \ldots \ldots 185$ Alladi Sitaram, On an analogue of the Wiener Tauberian theorem for symmetric spaces of the noncompact type 